\title{
RELAÇÃO ENTRE A DURAÇÃO DO ATO DA INSEMINAÇÃO ARTIFICIAL EM BOVINOS E A FERTILIDADE
}

\section{(Relationship between the lenght of the artifitial insemination act in bovine and the fertility)}

\author{
VENDRUSCOLO, M. '; KOZICKI, L.E. ${ }^{2}$; SEGUI, M.S. ${ }^{3}$; WEISS, R.R. ${ }^{4}$; SANTOS, I.V. ${ }^{4}$ \\ ${ }^{1}$ Médica veterinária; \\ ²Pontifícia Universidade Católica do Paraná, PUCPR e Universidade Federal do Paraná, UFPR; \\ ${ }^{3}$ Pontifícia Universidade Católica do Paraná, PUCPR; \\ ${ }^{4}$ Universidade Federal do Paraná, UFPR.
}

RESUMO - O presente experimento objetivou estudar a velocidade, em termos de tempo dispendido, à execução da inseminação artificial (IA) propriamente dita, em bovinos de corte, correlacionandose com a fertilidade obtida nos animais pesquisados, bem como verificar a interferência da perícia dos inseminadores quando do ato da IA, além da interferência do conjunto de múltiplas variáveis (mês, período, tempo, número de partos, escore da condição corporal, touro,raça, temperatura) sobre a taxa de prenhez. Utilizou-se dois grupos de vacas da raça Nelore de Fazendas de Naviraí (MS), sendo o grupo I (=animais inseminados em período de tempo de até dois minutos) e o grupo II (= animais inseminados em período de tempo acima de dois minutos). As vacas foram inseminadas artificialmente uma única vez, com sêmen fértil e estavam clinicamente sadias. A detecção do estro era feita duas vezes /dia (manhã e tarde) mediante rufiões e acompanhada pelos inseminadores. A duração do ato inseminatório considerado foi o decorrido entre a secagem da palheta de sêmen, imediatamente após a descongelação, até a retirada do aplicador universal de sêmen com a bainha do trato genital da fêmea, após a deposição do sêmen no corpo uterino. O diagnostico de gestação foi executado 60 dias após a IA. Ao final concluiu-se que a perícia do inseminador ao executar o ato da IA propriamente dito, afeta significativamente a taxa de concepção e que o tempo dispendido à execução do ato da IA nos bovinos, interfere na taxa de concepção, ao ser analisado isoladamente $(p<0,05)$, e ao ser verificado em conjunto com outras variáveis, a diferença mostrou-se ao nível de $p<0,1$.

Palavras-chaves: Inseminação artificial, bovinos, fertilidade, duração da IA.

ABSTRACTS - The aim of the present experiment was to establish a relationship between the time consumed to perform artificial insemination (AI) and fertility in beef cows. Furthermore, an analysis has been carried out in regard to the inseminators dexterity and the interference of different variables on the number of pregnancies: month, period, time, number of parturitions, body condition, bull, race, temperature. Two groups of Nelore cows from Navirai farms (MS) were used in this experiment. Cows from group I were inseminated during a time no longer than two minutes and cows from group II above two minutes. All cows were healthy and were artificially inseminated only once, the semen being fertile. The detection of the oestrus was performed twice a day, in the morning and the afternoon, with ruffians and in the presence of the inseminators. The length of the insemination has been considered as the time between the drying of the semen pellet, immediately after being melted and its deposition in the uterine body followed by the withdrawal of the universal semen applicator with the sheath from the female genital tract. The diagnosis of gestation has been carried out 60 days after the Al. It has been concluded that the dexterity of the inseminator in carrying on the process itself of Al significantly affects the rate of conception. In regard to the length of time taken to perform $\mathrm{Al}$ in bovines it has been found that it interferes in the rate of conception when considered alone $(p<0.05)$, the difference, however, being of $p<0.1$, when considered together with the other variables.

Key-words: artificial insemination, beef cows, fertility, Al duration. 


\section{Introdução}

Segundo GUIMARÃES et al. (2002), a eficiência reprodutiva correlaciona-se negativamente com o número de inseminações e com o período de serviço.

Por outro lado GWAZDAUSKAS et al. (1980) relacionam a fertilidade à inseminação artificial (IA), podendo esta ser maximizada quando executada em vacas em estro, utilizando-se adequados procedimentos na descongelação do sêmen, depositando-se o sêmen no útero, massageando-se o útero e o clitóris após a inseminação e quando as vacas inseminadas são mantidas a temperaturas abaixo de $23^{\circ} \mathrm{C}$ no dia seguinte à inseminação.

As vantagens da utilização da $\mathrm{I} A$, apontadas pela ASBIA (2004), são o melhoramento genético rápido e eficiente. Quanto às limitações da prática da IA, VANZIN (2002), destaca que a natalidade do rebanho pode ser drasticamente reduzida se a técnica não for corretamente aplicada. DINIZ (1996) questiona o porque das falhas das inseminações artificiais. Segundo a Associação Brasileira de Inseminação Artificial, (ASBIA, 2003), os fatores que afetam os resultados da IA são: o manuseio e descongelação inadequada do sêmen, o estresse das matrizes, os problemas ginecológicos e clínicos, o manejo reprodutivo da fazenda, a baixa qualificação da mão de obra; a detecção inadequada do estro bem como o horário da inseminação e a falta de assistência técnica.

Segundo DINIZ (1996), de nada valem bom manejo, boa assistência técnica, e inseminador competente, se o sêmen aplicado não for de boa qualidade fecundante. É muito importante que se conheça a procedência deste sêmen, e que independente da origem, antes de se iniciar qualquer estação reprodutiva, seja realizada sua análise microscópica. VÁSQUEZ e HERRERA (2003), relatam que os glicocorticóides são conhecidos inibidores da produção de anticorpos, da fagocitose, da produção de fatores ativadores dos linfócitos e da produção de fatores de crescimento das células $t$, indicando sua elevada influência sobre o sistema imune. Nas condições em que os animais estão sendo submetidos nos sistemas modernos de produção, vêm-se provocando neles diferentes graus de estresse devidos a alterações nutricionais, patológicas, metabólicas e/ou ambientais, causando aumento nos níveis plasmáticos de glicocorticóides, principalmente o cortisol.

LENSINK (2002) relata que vários projetos de pesquisa, a maioria conduzida com animais mantidos sob condições intensivas, demonstraram que os contatos físicos qualificados como "negativos", como bater nos animais, induziram comportamentos de fuga dos animais em relação ao homem, o que geralmente foi interpretado como "medo". As respostas de medo depois de contatos "negativos" com o homem, podem não só causar comportamento de evitação nos animais, como também respostas fisiológicas de estresse.

PIMENTEL (1983) conclui que qualquer alteração na rotina dos animais como mudanças na temperatura, alimentação ou dor, provoca um desequilíbrio no organismo alterando as taxas de concepção.

Falhas na fertilização podem também ocorrer devido a anormalidades obstrutivas da porção tubular do sistema genital feminino (ZEMJANIS, 1980), podendo ser de natureza congênita. Esses defeitos impedem o encontro dos gametas masculino e feminino e quando bilaterais, determinam a esterilidade.

ALVAREZ (2003) destaca a presença de ovários estáticos, que é o termo usado para descrever ovários afuncionais. As vacas afetadas apresentam-se saudáveis, mas não apresentam ciclo ovariano. De acordo com HAFEZ e HAFEZ (2004) outro problema ginecológico que ocorre é a hipoplasia ovariana, na qual os animais afetados apresentam tratos reprodutivos infantis e não exibem sinais de estro.

Outro entrave à reprodução é o anestro pósparto, caracterizado por um período de ausência de manifestação estral, que quando prolongado, constitui causa determinante de baixa eficiência reprodutiva, segundo relatos de FERREIRA (1991), INSKEEP (1995) e JOLLY et al. (1995).

De acordo com RESENDE (2001), os sistemas inadequados de alojamento e manutenção das fêmeas em produção e reprodução prejudicam a manifestação dos sinais de estro. Segundo RESENDE (2001), as causas nutricionais são de grande relevância por afetar primeiramente todas as funções fisiológicas gerais do organismo animal e 
Relaçao entre a duração do ato da inseminação artificial em bovinos e a fertilidade

secundariamente refletindo-se em distúrbios no sistema reprodutivo.

Levando-se em consideração propriedades que fazem uso da IA, a perícia do inseminador afeta significativamente a taxa de concepção ao primeiro serviço (SENGER et al. 1981). VANZIN (2002), recomenda que o inseminador revise todo o processo de IA em um período de três a cinco anos, porquanto estes profissionais tendem a alterar alguns procedimentos com o passar do tempo. O inseminador é fundamental neste processo, pois de sua condição de trabalho, interesse, responsabilidade, preparo e higiene, dependerão, em grande parte, os resultados.

A insegurança do inseminador pode afetar o nível de fertilidade do rebanho, ao se referir aos inexperientes. Esta afirmação se baseia em dados que OLDS e SEATH (1954) coletaram, em relação aos inseminadores sem experiência. Deve-se ressaltar que a experiência aumenta a capacidade de inseminar.

A falha na identificação do estro é um dos grandes problemas em fazendas que utilizam a IA ou a monta controlada. Pouco adianta uma vaca bem nutrida e de boa fertilidade retornar ao estro logo após o parto, se o estro não for identificado.

Em função da interferência de vários fatores sobre a eficiência da IA, o presente estudo objetivou estudar a velocidade, em termos de tempo dispendido, à execução da inseminação artificial propriamente dita, em bovinos de corte, correlacionando-se com a fertilidade obtida nos animais pesquisados.

O presente experimento objetivou estudar a velocidade, em termos de tempo dispendido, à execução da inseminação artificial (IA) propriamente dita, em bovinos de corte, correlacionando-se com a fertilidade obtida nos animais pesquisados, bem como verificar a interferência da perícia dos inseminadores quando do ato da IA, além da interferência do conjunto de múltiplas variáveis (mês, período, tempo, numero de partos, escore da condição corporal, touro,raça, temperatura) sobre a taxa de prenhez.

\section{Material e Método}

O estudo foi conduzido em três fazendas de criação de bovinos de corte no Estado do Mato
Grosso do Sul, município de Naviraí. A latitude

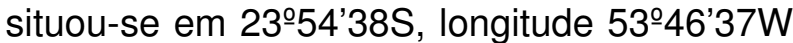
e altitude 362 metros, e temperatura média anual de $24^{\circ} \mathrm{C}$ e precipitação média anual de $1600 \mathrm{~mm}$. Durante os meses do experimento, de novembro de 2001 a fevereiro de 2002, a temperatura média foi de $24^{\circ} \mathrm{C}$ e a precipitação média mensal foi de $195 \mathrm{~mm}$, de acordo com dados do Instituto Nacional de Metereologia (INMET, 2004).

Os animais eram da raça Nelore, pluríparas, partos normais, sem complicações no pósparto, em bom estado sanitário, sendo o controle sanitário realizado com vermifugações e vacinações contra a brucelose, a febre aftosa e a clostridiose. Os animais encontravam-se com escore da condição corporal em torno de 3.5, de acordo com EDMONSON et al. (1989).

A idade dos animais variava entre 4 e 5 anos e o regime de criação era extensivo, permanecendo os animais durante todo o período do experimento em sistema de pasto, sendo a pastagem de boa qualidade e composta por Brachiaria decumbens, nas três propriedades. A técnica da IA era utilizada há três anos nas fazendas e os inseminadores eram suficientemente experientes e capazes.

Antes do experimento, fez-se a avaliação dos animais, quanto à taxa de parição do ano anterior, seleção das matrizes, avaliação do sêmen por partida para verificação da viabilidade espermática. Os animais foram divididos em dois grupos:

Grupo I: animais inseminados em período de tempo de até dois minutos (=2 minutos);

Grupo II: animais inseminados em período de tempo acima de dois minutos ( $>2$ minutos) (TABELA 1).

Foram analisados dados de 244 animais procedentes de três fazendas, sendo 50 animais do grupo I e 194 animais do grupo II. As vacas foram inseminadas uma única vez, com sêmen comprovadamente fértil. A detecção do estro, nos animais era feita duas vezes ao dia, sendo pela manhã, entre 7:30 e 8:30 horas e à tarde entre 17:00 e 18:00; os animais que encontravam-se em estro à tarde eram inseminados na manhã seguinte entre 7:00 e 7:30 horas e os detectados em estro pela manhã eram inseminados no final da tarde do mesmo dia, entre 18:00 e 19:00 horas. 
TABELA 1 - DIVISÃO DOS ANIMAIS, EM GRUPOS, DE ACORDO COM O TEMPO COM QUE O ATO INSEMINATÓRIO FOI EFETUADO NAS FAZENDAS 1, 2 E 3.

\begin{tabular}{cccccc}
\hline Fazendas & $\begin{array}{c}\text { Grupo I -IA em } \\
\text { até 2 minutos }\end{array}$ & $\begin{array}{c}\text { Percentual } \\
\text { grupo I }\end{array}$ & $\begin{array}{c}\text { Grupo II -IA } \\
\text { acima de } \\
\text { 2minutos }\end{array}$ & $\begin{array}{c}\text { Percentual } \\
\text { grupo II }\end{array}$ & $\begin{array}{c}\text { Total de } \\
\text { animais }\end{array}$ \\
\hline 1 & 32 & 40,5 & 47 & 59,5 & 79 \\
2 & 15 & 16,85 & 74 & 83,15 & 89 \\
3 & 3 & 4,1 & 73 & 95,9 & 76 \\
Total & 50 & 20,5 & 194 & 79,5 & 244 \\
\hline
\end{tabular}

O manejo reprodutivo consistiu na realização de exame ginecológico e na preparação dos rufiões. Estes possuíam pelagem diferenciada dos demais animais, boa libido, peso variando de 350 a $450 \mathrm{Kg}$ e temperamento dócil. As técnicas cirúrgicas utilizadas, para se fazer os rufiões, foram a caudectomia do epidídimo e a aderência cirúrgica do "S" peniano, sendo utilizado um rufião para cada grupo de 40 fêmeas.

Manejo na inseminação artificial:

O material foi organizado de maneira a permanecer próximo às vacas que seriam inseminadas no brete. As luvas foram calçadas pelo lado avesso e a descongelação do sêmen foi efetuada em água a $35^{\circ} \mathrm{C}$, por 30 segundos, quando se empregou a palheta de $0,5 \mathrm{ml}$ e por 20 segundos quando a palheta era de $0,25 \mathrm{ml}$. Igualmente a partir deste momento foram observados os procedimentos técnicos como a secagem da palheta, deslocamento da bolha de ar em direção ao lacre, corte da extremidade e encaixe na bainha e montagem do aplicador. No momento da IA evitou-se ao máximo o estresse sobre as fêmeas, ao se praticar o ato inseminatório. Havia uma pessoa para auxiliar no trabalho de limpeza vulvar, abertura dos lábios vulvares e apreensão da cauda. A seguir executou-se a passagem do aplicador pela cérvice, deposição do sêmen no corpo uterino e retirada do cateter.

O tempo do ato inseminatório considerado, foi o decorrido entre a secagem da palheta, imediatamente após a descongelação, até a retirada do aplicador universal de sêmen com a bainha do trato genital da fêmea, após a deposição do sêmen no corpo uterino. A seguir efetuou-se a massagem clitoriana vulvar e a marcação dos animais inseminados. O diagnóstico de gestação por toque retal, foi realizado 60 dias após a última IA.

Análise estatística:

Os cálculos estatísticos basearam-se no Teste de Qui-quadrado e no Teste Exato de Fischer, ambos para detectar a significância estatística da diferença entre os dois grupos (CAVALLI-SFORZA, 1974; MONTGOMERY, 1991 e SOFTWARE R, 2002). O software R é uma linguagem e ambiente para computação estatística e gráficos. É um projeto "General Public License" (GNU) similar à linguagem e ambiente $S$ desenvolvida no Bell Laboratories, disponibilizando grande variedade de métodos estatísticos e técnicas gráficas. Como as IA foram realizadas em cada fazenda por um inseminador, verificouse a possibilidade de haver diferença entre as fazendas (mais especificamente aos inseminadores) no tocante à eficiência dos mesmos; para tanto fez-se a comparação entre as fazendas, considerando-se o número de animais gestantes em cada uma, sem considerar a divisão do tempo (até dois minutos e acima de dois minutos). Para esta variável utilizou-se o Teste do Qui-quadrado e o Teste Exato de Fischer, ambos para detectar diferença estatística entre as fazendas, ou seja, diferença entre eficiência da mão-de-obra empregada em cada fazenda (CAVALLI-SFORZA, 1974; MONTGOMERY, 1991 e SOFTWARE R, 2002). Assim sendo foi realizada outra análise, utilizando uma técnica conhecida como Modelos Lineares Generalizados. Diversas variáveis foram analisadas, no sentido de proporcionar maior confiabilidade aos resultados (SOFTWARE $R, 2002$ ). Variáveis estudadas: tempo necessário para execução da IA propriamente dita, até dois minutos e acima de dois minutos; perícia dos inseminadores de cada fazenda; época das IA (estação reprodutiva de novembro a 
fevereiro); o touro doador do sêmen; o período da execução das IA (pela manhã ou à tarde); a raça dos touros; o escore da condição corporal de cada fêmea no dia da IA; o número de partos dos animais e a localização das fazendas (município), temperatura média mensal, latitude, altitude e precipitação pluviométrica mensal, segundo o INMET (2004).

\section{Resultados e Discussão}

Na TABELA 2 são expostos os resultados obtidos, no diagnóstico de gestação, de vacas da raça nelore, ao se comparar os tempos gastos pelos inseminadores, para realizar a IA, especificamente à rapidez na execução do ato inseminatório propriamente dito.

TABELA 2 - ACHADOS DO DIAGNÓSTICO DE GESTAÇAO, DE VACAS DA RAÇA NELORE, POR PALPAÇÃO RETAL, REALIZADO 60 DIAS APÓS AÚLTIMA INSEMINAÇÃO ARTIFICIAL. MATO GROSSO DO SUL, 2002. TOTAL (N=244).

\begin{tabular}{cccccc}
\hline Grupos & $\begin{array}{c}\text { No de vacas } \\
\text { Prenhes }\end{array}$ & $\begin{array}{c}(\%) \text { de vacas } \\
\text { prenhes }\end{array}$ & $\begin{array}{c}\text { № de vacas } \\
\text { não prenhes }\end{array}$ & $\begin{array}{c}(\%) \text { de vacas } \\
\text { não prenhes }\end{array}$ & Total \\
\hline I - até 2 $\min$ & 42 & 84,0 a & 8 & 16,0 & 50 \\
II - acima de 2 min & 117 & $65,5 b$ & 77 & 34,5 & 194 \\
\hline Total & 149 & & 85 & & 244 \\
\hline
\end{tabular}

$a: b P<0,05$

Confrontando os dados obtidos no presente experimento, observou-se que quando a variável tempo foi analisada isoladamente e dividida em dois grupos, grupo I (IA em até dois minutos e grupo II acima de dois minutos), a velocidade com que se realizou a IA nas vacas, influenciou a taxa de prenhez. Observa-se na TABELA 2, significativa diferença $(p<0,05)$ na taxa de fertilidade nos animais que foram inseminados em até dois minutos $(84,0 \%)$, contra $(65,5 \%)$ dos animais inseminados com o tempo superior a dois minutos. A análise estatística evidenciou que a probabilidade dos animais tornarem-se prenhes através de uma inseminação artificial realizada em até dois minutos é 3,46 vezes maior do que quando realizada em mais que dois minutos.

TABELA 3 - VALORES ENCONTRADOS, NO DIAGNÓSTICO DE GESTAÇAO, DE VACAS DA RAÇA NELORE INSEMINADAS EM ATÉ DOIS MINUTOS (GRUPO I) E ACIMA DE DOIS MINUTOS (GRUPO II), POR PALPAÇÃO RETAL, REALIZADO 60 DIAS APÓS A ÚLTIMA INSEMINAÇÃO ARTIFICIAL. MATO GROSSO DO SUL, 2002.

\begin{tabular}{|c|c|c|c|c|c|c|}
\hline Grupo & Fazenda & $\begin{array}{l}\text { № vacas } \\
\text { prenhes }\end{array}$ & $\begin{array}{c}\text { (\%) vacas } \\
\text { prenhes }\end{array}$ & $\begin{array}{c}\text { № vacas } \\
\text { não } \\
\text { prenhes }\end{array}$ & $\begin{array}{c}\text { (\%) vacas } \\
\text { não } \\
\text { prenhes }\end{array}$ & Total \\
\hline I & 1 & 26 & 81,25 & 6 & 18,75 & 32 \\
\hline I & 2 & 14 & 93,3 & 1 & 6,7 & 15 \\
\hline I & 3 & 2 & 66,7 & 1 & 33,3 & 3 \\
\hline Total grupo I & & 42 & & 8 & & 50 \\
\hline II & 1 & 31 & 65,95 & 16 & 34,05 & 47 \\
\hline II & 2 & 57 & 77 & 17 & 23 & 74 \\
\hline II & 3 & 29 & 39,7 & 44 & 60,3 & 73 \\
\hline Total grupo II & & 117 & & 77 & & 194 \\
\hline Total & & 159 & & 85 & & 244 \\
\hline
\end{tabular}


Quando os dados foram analisados, sob o aspecto intra-fazenda, (TABELA 3), (fazendas 1,2 e 3 isoladamente), não detectou-se diferença, ou seja, o tempo de IA não interferiu na fertilidade do rebanho, fato este que pode ser explicado em função da baixa freqüência de dados em cada fazenda. Quando os dados das três fazendas foram somados e analisados conjuntamente, a probabilidade dos animais tornarem-se prenhes através de inseminação artificial em até dois minutos foi 3,46 vezes maior, do que nos animais cuja IA foi realizada em mais que dois minutos, (TABELA 3 ).

Neste ínterim foi verificado a possibilidade de haver diferença entre as fazendas, quanto à eficiência dos inseminadores, ou seja se haveria interferência da perícia dos inseminadores sobre a fertilidade do rebanho. Nas TABELAS 4, 5 e 6 são apresentados os valores encontrados no diagnóstico de gestação, confrontando-se os dados das fazendas entre si.

TABELA 4 - VALORES ENCONTRADOS, NO DIAGNÓSTICO DE GESTAÇAO, DE VACAS DA RAÇA NELORE, FAZENDAS $1 \mathrm{E} 2$, POR PALPAÇÃO RETAL, REALIZADO 60 DIAS APÓS A ÚLTIMA INSEMINAÇÃO ARTIFICIAL. MATO GROSSO DO SUL, 2002.

\begin{tabular}{cccccc}
\hline Grupos & $\begin{array}{c}\text { № vacas } \\
\text { prenhes }\end{array}$ & $\begin{array}{c}\text { \% vacas } \\
\text { prenhes }\end{array}$ & $\begin{array}{c}\text { No vacas não } \\
\text { prenhes }\end{array}$ & $\begin{array}{c}\text { \% vacas não } \\
\text { prenhes }\end{array}$ & Total \\
\hline Fazenda 1 & 57 & 72,1 & 22 & 27,9 & 79 \\
Fazenda 2 & 71 & 79,8 & 18 & 20,2 & 89 \\
\hline Total & 128 & & 40 & & 168
\end{tabular}

Quando analisou-se os dados das fazendas 1 e 2, (possibilidade de haver diferença entre fazendas), quanto à perícia dos inseminadores influenciando a fertilidade, não foram detectadas diferenças, ou seja , a mão de obra não diferiu entre as fazendas 1 e 2 , (TABELA 4).

TABELA 5 - VALORES ENCONTRADOS, NO DIAGNÓSTICO DE GESTAÇAO, DE VACAS DA RAÇA NELORE, FAZENDAS $1 \mathrm{E} 3$, POR PALPAÇÃO RETAL, REALIZADO 60 DIAS APÓS A ÚLTIMA INSEMINAÇÃO ARTIFICIAL. MATO GROSSO DO SUL, 2002.

\begin{tabular}{cccccc}
\hline Grupos & $\begin{array}{c}\text { No vacas } \\
\text { prenhes }\end{array}$ & $\begin{array}{c}\text { \% vacas } \\
\text { prenhes }\end{array}$ & $\begin{array}{c}\text { №vacas não } \\
\text { prenhes }\end{array}$ & $\begin{array}{c}\text { \% vacas não } \\
\text { prenhes }\end{array}$ & Total \\
\hline Fazenda 1 & 57 & $72,1 \mathrm{a}$ & 22 & 27,9 & 79 \\
Fazenda 3 & 31 & $40,8 \mathrm{~b}$ & 45 & 59,2 & 76 \\
\hline Total & 88 & & 67 & & 155 \\
\hline
\end{tabular}

$$
P<0,05, a: b
$$

Por outro lado ao se confrontar os dados das fazendas 1 e 3 , verificou-se diferença $(p<0,05)$, entre a perícia dos inseminadores, de tais fazendas, quanto ao ato de inseminação e a fertilidade dos animais. A possibilidade de os animais tornarem-se prenhes através de inseminação artificial realizada por inseminador da fazenda 1 , foi 3,76 vezes maior do que quando inseminados na fazenda 3, (TABELA 5).

Ao se comparar os dados das fazendas 2 e 3, (TABELA 6), houve igualmente diferença entre estas fazendas. A probabilidade dos animais serem fertilizados através de inseminação artificial realizada na fazenda 2 , foi 5,73 vezes maior do que quando inseminados na fazenda 3. 
Relaçao entre a duração do ato da inseminação artificial em bovinos e a fertilidade

TABELA 6 - VALORES ENCONTRADOS, NO DIAGNÓSTICO DE GESTAÇAO, DE VACAS DA RAÇA NELORE, FAZENDAS 2 E 3, REALIZADO 60 DIAS APÓS A ÚLTIMA INSEMINAÇÃO ARTIFICIAL. MATO GROSSO DO SUL, 2002.

\begin{tabular}{cccccc}
\hline Grupos & $\begin{array}{c}\text { No vacas } \\
\text { prenhes }\end{array}$ & $\begin{array}{c}\text { \% vacas } \\
\text { prenhes }\end{array}$ & $\begin{array}{c}\text { № vacas não } \\
\text { prenhes }\end{array}$ & $\begin{array}{c}\text { \% vacas não } \\
\text { prenhes }\end{array}$ & Total \\
\hline Fazenda 2 & 71 & $79,8 \mathrm{a}$ & 18 & 20,2 & 89 \\
Fazenda 3 & 31 & $40,8 \mathrm{~b}$ & 45 & 59,2 & 76 \\
\hline Total & 102 & & 73 & & 165 \\
\hline
\end{tabular}

$\mathrm{P}<0,05 \mathrm{a}: \mathrm{b}$

Ao se comparar os dados das fazendas 2 e 3, (TABELA 6), houve igualmente diferença entre estas fazendas. A probabilidade dos animais serem fertilizados através de inseminação artificial realizada na fazenda 2, foi 5,73 vezes maior do que quando inseminados na fazenda 3 .

Ao se testar a perícia dos inseminadores, e correlacioná-la ao número de vacas prenhes nas três fazendas, observou-se que os resultados relativos à eficiência da mão de obra utilizada, na fazenda 3 , diferiu dos obtidos nas fazendas 1 e 2, ou seja a taxa de prenhez, obtida na fazenda 3 foi menor que a das demais fazendas. Deve-se ressaltar que foi na fazenda 3 , que houve maior porcentagem de animais inseminados em período de tempo superior a dois minutos, (96\% dos animais inseminados), tornando essa observação indicativa de que quanto maior o tempo da IA, menor é a probabilidade de gestação.

$O$ fato de que 0 inseminador da fazenda 3 tenha demorado mais tempo para a execução do ato da IA nas vacas e conseqüentemente ter obtido a taxa de prenhez menor, pode corroborar afirmação de CORRÊA (2003), em função de reações às agressões involuntárias e localizadas da ponta da pipeta, com muitas tentativas improdutivas de passagem da mesma, via cérvice.

Realizadas as análises das variáveis tempo de IA e inseminadores interferindo sobre a fertilidade dos grupos, executou-se outra análise estatística, utilizando-se os Modelos Lineares Generalizados, (SOFTWARE R, 2002), na tentativa de verificar a possível interferência de outros fatores (mês, período, tempo, touro, raça, escore da condição corporal, número de partos, local, temperatura, latitude, longitude, altitude e precipitação), sobre a fertilidade do rebanho, fixando-se a taxa de prenhez como variável resposta. Verificou-se que a variável tempo influenciou a fertilidade, ao nível de $90 \%$ de confiança, $(p<0,1)$. Muito embora a diferença estatística ao nível de $10 \%$ seja elevada, poder-se-ia inferir, que se houvesse maior número de vacas envolvidas no experimento, essa diferença poderia tornar-se menor e conseqüentemente ser significativa em níveis estatísticos aceitáveis.

Faz-se necessário ressaltar que a literatura disponível a respeito da velocidade do tempo de IA executado em vacas influenciando a taxa de prenhez, mostrou-se inexistente. Em função desse fato a discussão do presente trabalho aparentemente tornou-se prejudicada. Sugerese que idêntica sistemática de futuros experimentos sejam conduzidas, no sentido de se aumentar o número de animais envolvidos.

Ao final das análises realizadas pode-se concluir que:

1 - A perícia do inseminador ao executar o ato da inseminação artificial propriamente dito, afeta significativamente a taxa de concepção à primeira inseminação artificial.

2 - O tempo necessário para a execução do ato da inseminação artificial nos bovinos, interfere na taxa de concepção, ao ser analisado isoladamente, $(p<0,05)$, e quando analisado em conjunto com outras variáveis a diferença mostrou-se ao nível de $(p<0,1)$.

\section{REFERÊNCIAS}

\section{ALVAREZ; R.H. Problemas reprodutivos no pós- parto. 2003. Disponível em: <http:// www.snagricultura.org.br/artigos/artitec- bovinos01.htm>. Acesso em: 10 fev. 2004.}

ASBIA (2003). Associação Brasileira de Inseminação Artificial. Disponível em: <www.asbia.org.br>. Acesso em: 10 set. 2003.

ASBIA (2004). Associação Brasileira de Inseminação Artificial. Disponível em: <www.asbia.org.br>. Acesso em: 19 mai. 2004. 
CAVALLI - SFORZA, L. Biometrie: Grundziege biologisch - Medizinescher Statistik. Verlag: Gustav Fischer, 1974. p. 55-58.

CORRÊA, L. Inflamação. Faculdade de Odontologia da USP. Disponível em: <www.usp.br/ fo>. Acesso em: 23 abr. 2003.

DINIZ, O. Inseminação artificial. Pardo-suíço em Revista, maio/jun. 1996. Disponível em: $<w w w . p a r d o-s u i c o . c o m . b r / i n s e m . h t m>$. Acesso em: 02 abr. 2003.

EDMONSON, A.J.; LEAN, I.J.; WEAVER, L.D. A body condition scoring chat for Holstein dairy cows. Journal of Dairy Science, Savoy, v. 72, p. 68-78, 1989.

FERREIRA, A.M. Redução do período de serviço em taurinos. In: CONGRESSO BRASILEIRO DE REPRODUÇÃO ANIMAL, 1991, Belo Horizonte. Anais... Belo Horizonte: Colégio Brasileiro de Reprodução Animal,1991. v.9, p.22-32.

GUIMARÃES, J.D.; ALVES, N.G.; COSTA, E.P.; SILVA, M.R.; COSTA, F.M.J.; ZAMPERLINI, B. Eficiências reprodutivas e produtivas em vacas das raças gir, holandês e cruzadas holandês $x$ zebu. Revista Brasileira de Zootecnia, Viçosa, v. 31, n 2. p. 641-647 2002.

GWAZDAUSKAS, F.C.; LINEWEAVER, J.A.; VINSON, W.E. Rates of conception by artificial insemination of dairy cattle. Journal of Dairy Science, Savoy, v. 64, p. 358, 1980.

HAFEZ, E.S.E.; HAFEZ,B. Reprodução animal. 7. ed. São Paulo: Manole, 2004. p. 431-467.

INSKEEP, E.K. Factors that affect fertility during oestrus cycles with short or normal luteal phases in post partiem cows. Journal Reproduction and Fertility, Cambridge, v. 49, suppl., p. 493-503, 1995.

INSTITUTO NACIONAL DE METEREOLOGIA, 2004. Disponível em: <www.inmet.gov.br/ climatologia/combo_climatologia_C.html>. Acesso em: 24 maio 2004.

JOLLY, P.D.; MCDOUGALL, S.; FITZRTRICK, L.A. et al. Physiological effects of undernutrition on postpartum anoestrus in cows. Journal Reproductive and Fertility, Cambridge, v. 49, suppl., p. 492, 1995.

Recebido para publicação: 25/04/2005

Aprovado:

20/09/2005
LENSINK. B.J. A relação homem-animal na produção animal. I Congresso virtual global sobre produção orgânica de bovinos de corte.Via internet, disponível em: http://www.bovinos.ufc.br/ prodanimal.pdf. Acesso em: 05 de abr 2004, editado pela Universidade do Contestado- Concórdia- SC, EMBRAPA Pantanal- Corumbá-MS, set. 2002.

MONTGOMERY, D. Desing and analysis of experiments. 3. ed. New York, p.27-37, 1991.

OLDS, D.; SEATH, D.M. Factors affectin reproductive efficiency in dairy cattle. Kentucky Agriculture Experiment Station Bulletin, Lexington, 605, 1954.

PIMENTEL, C.A. Repetição de serviço - "Repeat breeding". In: SIMPÓSIO NACIONAL DE REPRODUÇÃO ANIMAL, 5, 1983, Belo Horizonte. Anais... v.5, p.17-36, 1983.

RESENDE, O.A. Problemas não infecciosos que afetam a reprodução de bovinos? Visão do veterinário de campo. Revista Brasileira de Reprodução Animal, Belo Horizonte, v. 25, n. 2, 2001.

SENGER, P.L.; HILLERS, J.K.; MITCBELL, J.R.; FLEMING, W.N.; DARLINGTON, R.L. Research summary of factors affecting conception to first service in dairy cows. Part I - Bulls inseminators and semen quality. Proceedings of the Annual Meeting Society of Therionelogy, Washington, $p$. 126-134, 1981.

SOFTWARE R. Copyright 2002, The R development core team- version 1.5 .0 patched (06/ 12/2002). Software livre, acesso gratuito.

VANZIN, I.M. Manual de Inseminação Artificial Pecplan Bradesco, "on line" Disponível em: <http:// www.pecplanabs.com.br>. Acesso em: 10 out. 2002a.

VASQUEZ, E.F.A.; HERRERA, A.P.N. Concentração plasmática de cortisol, uréia, cálcio e fósforo em vacas de corte mantidas a pasto suplementadas com levedura de cromo durante a estação de monta. Ciência Rural, Santa Maria, v. 33, n. 4, p. 743-747, 2003.

ZEMJANIS, R. Repeat - breeding or conception failure in cattle. In: MORROW, D.A. Current therapy in theriogenology. Philadelphia: W. B. Saunders, 1980. p. 205-213. 